\title{
miR-552 promotes ovarian cancer progression by regulating PTEN pathway
}

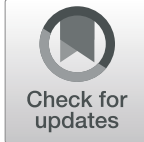

Wenman Zhao ${ }^{1^{* \dagger}}$, Tao Han ${ }^{2 \dagger}$, Bao Li ${ }^{1 \dagger}$, Qianyun $\mathrm{Ma}^{3}$, Pinghua Yang ${ }^{4^{*}}$ and Hengyu $\mathrm{Li}^{5^{*}}$ (D

\begin{abstract}
Background: Increasing researches have demonstrated the critical functions of MicroRNAs (miRNAs) in the progression of malignant tumors, including ovarian cancer. It was reported that miR-552 was an important oncogene in both breast cancer and colorectal cancer. However, the role of miR-552 in ovarian cancer (OC) remains to be elucidated.

Methods: RT-PCR and western blot analysis were used to detect the expression of miR-552 and PTEN. The impact of miR-552 on ovarian cancer proliferation and metastasis was investigated in vitro. The prognostic value of miR552 was evaluated using the online bioinformatics tool Kaplan-Meier plotter.

Results: In the present study, we for first found that miR-552 was upregulated in ovarian cancer, especially in metastatic and recurrence ovarian cancer. Forced miR-552 expression promotes the growth and metastasis of ovarian cancer cells. Consistently, miR-552 interference inhibits the proliferation and metastasis of ovarian cancer cells. Mechanically, bioinformatics and luciferase reporter analysis identified Phosphatase and tension homolog (PTEN) as a direct target of miR-552. miR-552 downregulated the PTEN mRNA and protein expression in ovarian cancer cells. Furthermore, the PTEN siRNA abolishes the discrepancy of growth and metastasis capacity between miR-552 mimic ovarian cells and control cells. More importantly, upregulation of miR-552 predicts the poor prognosis of ovarian cancer patients.
\end{abstract}

Conclusion: Our findings revealed that miR-552 could promote ovarian cancer cells progression by targeting PTEN signaling and might therefore be useful to predict patient prognosis.

Keywords: Ovarian cancer, miR-552, PTEN, Proliferation, Metastasis

\section{Introduction}

Ovarian cancer is one of the most deadly gynecological malignances in the world, causing more than 140,000 deaths yearly $[1,2]$. Early detection of ovarian cancer patients with a five-year survival rate is about 90\% [3]. However, about 70\% ovarian cancer patients were diagnosis at advanced stage and have a poor prognosis [4]. Although as the surgery, chemotherapy and radiotherapy progress,

\footnotetext{
*Correspondence: cxrmyyzwm@126.com; yangpinghua2008@163.com; Ihy@smmu.edu.cn

'Wenman Zhao, Tao Han and Bao Li contributed equally to this work. 'Department of General surgery, Cao county people's hospital, East of Qinghe Road, Heze 274400, Shandong province, China

${ }^{4}$ Department of Biliary Tract Surgery, Third Affiliated Hospital of Second Military Medical University, Shanghai 200438, China

${ }^{5}$ Department of Breast and Thyroid surgery, First Affiliated Hospital of Second Military Medical University, Shanghai 200433, China

Full list of author information is available at the end of the article
}

the overall ovarian cancer survival rate has not improved in the past decades $[5,6]$. Therefore, it's urgent to research the underlying mechanism of ovarian cancer and find the new therapeutic target to improving the clinical outcome of patients suffering from ovarian cancer.

MicroRNAs (miRNAs) are a class small non-coding RNA molecule, containing about 22 nucleotides, found in plants, animals and some viruses, which are capable of regulating gene expression at both the transcriptional and translational levels miRNAs [7, 8]. Accumulating evidence indicates that miRNAs play important functions in various biological processes, including cell proliferation, apoptosis, differentiation and migration $[9,10]$. More researches also find that miRNAs modulate the proliferation, apoptosis, metastasis and metabolism of various cancers, including

(c) The Author(s). 2019 Open Access This article is distributed under the terms of the Creative Commons Attribution 4.0 International License (http://creativecommons.org/licenses/by/4.0/), which permits unrestricted use, distribution, and reproduction in any medium, provided you give appropriate credit to the original author(s) and the source, provide a link to the Creative Commons license, and indicate if changes were made. The Creative Commons Public Domain Dedication waiver (http://creativecommons.org/publicdomain/zero/1.0/) applies to the data made available in this article, unless otherwise stated. 
breast cancer, lung cancer, liver cancer and ovarian cancer [11-13]. These previous studies remind us that miRNAs may also serve as potential prognosis biomarkers and a novel therapeutic target in various cancers $[14,15]$.

miR-552 is a newly discovered miRNA, its function and mechanism of action in biological processes and diseases are not completely understood. Previous studies showed that miR-552 promotes colorectal cancer cells proliferation and migration by directly targeting $\mathrm{DACH} 1$ via the $\mathrm{Wnt} / \beta$-catenin signaling pathway [16]. Moreover, miR-552 also enhances metastatic capacity of colorectal cancer cells by targeting a disintegrin and metalloprotease 28 [17]. However, the role of miR-552 in ovarian cancer was little known.

The results of the present study demonstrate that miR-552 was upregulated in ovarian cancer, and that elevated expression of miR-552 was correlated with poor patient prognosis. In addition, we found that miR-552, acting as an oncogene, was involved in ovarian cancer progression, and that miR-552 dramatically facilitated ovarian cancer cell proliferation and metastasis. Notably, it was demonstrated that PTEN was a direct target of miR-552, the levels of which decreased when miR-552 was overexpressed. Our results highlight the importance of miR-552 in promoting the proliferation and metastasis of ovarian cancer cells.

\section{Materials and methods}

\section{Tissue samples}

In total, 80 cases of tumor tissues and their corresponding adjacent noncancerous tissues were obtained from ovarian cancer patients in Cao county people's hospital from 2012 to 2017, detailed clinicopathological features of the patients is described in online Additional file 1: Table S1. Fifteen cases of tumor tissues and their metastases tissues were obtained from ovarian cancer patients in Cao county people's hospital from 2013 to 2016. Fifteen cases of tumor tissues and their recurrence tumor tissues were obtained from ovarian cancer patients in Cao county people's hospital from October 2014 to March 2016. Human ovarian cancer and adjacent normal tissues were immediately snap-frozen in liquid nitrogen and stored at $-80^{\circ} \mathrm{C}$. All of the patients provided signed informed consent. The medical ethics committee of Cao county people's hospital approved the retrieval method for cancer specimens.

\section{Cell lines and cell culture}

HO8910 and HGSOC cells were purchased from Chinese Academy of Sciences, Shanghai, China. The ovarian cancer cells were cultured in RPMI-1640 medium supplemented with $10 \%$ fetal calf serum
(FCS; Invitrogen, Carlsbad, CA, USA). The cultures were dissociated with $0.5 \%$ trypsin and transferred to new six-well plates biweekly. The lenti-vector expressing miR-552 sponge or miR-552 mimic and their control virus were purchased from Shanghai GenePharma (Shanghai, China). LV3-has-miR-552 mimics (5' - AACAGGTGACTGGTTAGACAA - 3') can mimic high levels of endogenous mature miR552 in cells. The miR-552 sequence +loop+ reverse complementation (incomplete complementation) was inserted into the vector to express shRNA, which was then cut into mature miR-552. LV3-has-miR-552 sponge $\left(5^{\prime}\right.$ - TTGTCTAACCAGTCACCTGTT - $\left.3^{\prime}\right)$ is that the complete reverse complementary sequence (inhibitor) of miRNA, linked by linker, which adsorbs the binding miRNA (it may adsorb but not degrade, or it may lead to degradation of miRNA), resulting in miRNA not functioning biologically. LV3-has-miR-552 mimics and LV3-has-miR-552 sponge were packaged as a lentivirus. The lentivirus titers are about $1 \times 10^{8} \mathrm{TU} / \mathrm{ml}$. HO8910 and HGSOC cells were seeded into 6 -well plate and then added $100 \mu \mathrm{l}$ lentivirus. The stable infectants were screening by using puromycin [18].

HO8910 and HGSOC cells were seeded into a six-well plate until they reached $60-70 \%$ confluence. Transfection of si-PTEN or its negative control was performed in each well in the absence of serum with siRNA transfection reagent according to the manufacturer's instructions (Polyplus, Illkirch, France). The sequence of si-PTEN is as follows: 5' - CCACAGCUAGAACUUAUCAAATT 3'. This siRNA interrupt PTEN transcript variant 1and transcript variant 2. The siRNA was purchased from Shanghai GenePharma (Shanghai, China).

\section{Cell proliferation assays}

For cell proliferation analysis, ovarian cancer cells were seeded in 96-well plates $\left(3 \times 10^{3}\right.$ cells per well). ATP activity was measured using a Cell Counting Kit- 8 at indicated time points. ATP activity was measured using a Cell Counting Kit- 8 at indicated time points. The procedure was as follows: The cell suspension $(100 \mu \mathrm{l} /$ well $)$ was inoculated in a 96-well plate, and the plate was pre-incubated in a humidified incubator at $37^{\circ} \mathrm{C}$ for $1 \mathrm{~h}$. This was followed by the addition of $10 \mu \mathrm{l}$ of the CCK- 8 solution to each well of the plate, and incubation of the plate for $1 \mathrm{~h}$ in the incubator. Finally, the absorbance was measured at $450 \mathrm{~nm}$ using a microplate reader (Synergy H1; BioTek Instruments, Inc., Winooski, VT, USA) [19].

\section{Colony formation assay}

For colony formation assay, ovarian cancer cells were cultured in 12-well plates $\left(3 \times 10^{3}\right.$ cells/well). The cells were 
incubated at $37^{\circ} \mathrm{C}$ for 7 days and then fixed with with $10 \%$ neutral formalin for $>4 \mathrm{~h}$. The cells were dyed with crystal violet (Beyotime, Haimen, China). The cells were photographed under a microscope (Olympus, Tokyo, Japan).

\section{EdU immunofluorescence staining}

For cell EdU immunofluorescence staining, ovarian cancer cells were seeded into 96-well plates and performed using the EdU Kit (RiboBio). The results were quantified with a Zeiss axiophot photomicroscope (Carl Zeiss) and Image-Pro plus 6.0 software.

\section{Cell migration assays}

For cell migration experiments, $2 \times 10^{5}$ ovarian cancer cells were seeded into the upper chamber of a polycarbonate transwell in serum-free RPMI-1640 medium. The lower chamber was added with RPMI-1640 medium containing 20\% FBS as chemoattractant. The cells were incubating for $12 \mathrm{~h}$ and the chamber was fixed with $10 \%$ neutral formalin for $>4 \mathrm{~h}$. The cells were dyed with crystal violet (Beyotime). The cells were then counted under a microscope (Olympus) and the cell number is expressed as the average number of the cells in each field.

\section{Cell invasion assays}

For cell invasion experiments, $2 \times 10^{5}$ ovarian cells were seeded into the upper chamber of a polycarbonate transwell in serum-free RPMI-1640 medium. The lower chamber was added with RPMI-1640 medium containing 20\% FBS as chemoattractant. The cells were incubating for $24 \mathrm{~h}$ and the chamber was fixed with $10 \%$ neutral formalin for $>4 \mathrm{~h}$. The cells were dyed with crystal violet (Beyotime). The cells were

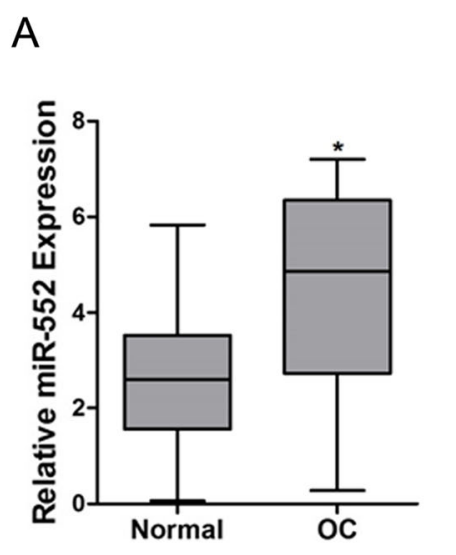

B

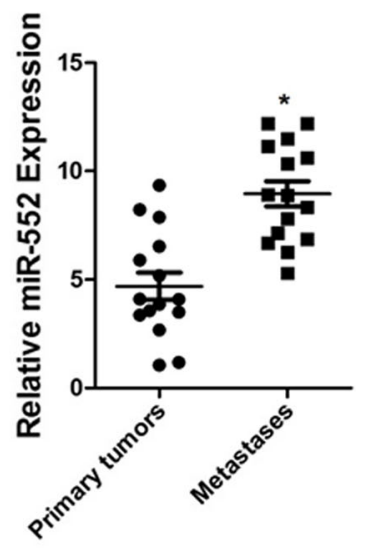

C

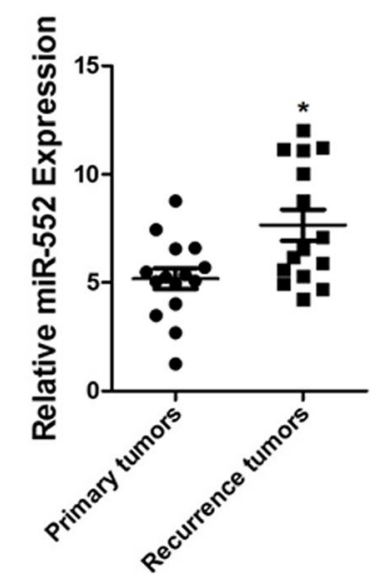

D

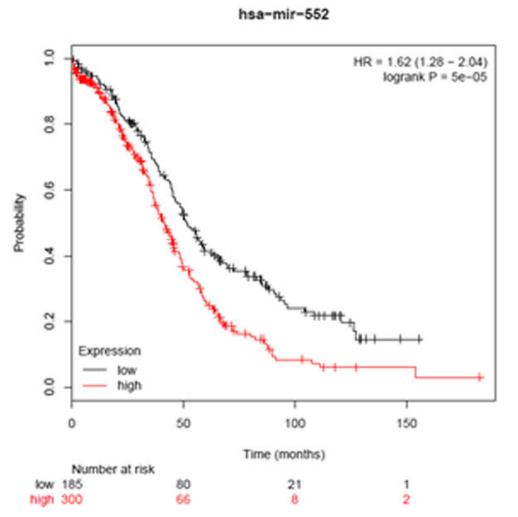

Fig. 1 Expression of miR-552 in human OC tissues. a. The expression of miR-552 in 80 pairs of ovarian cancer (T) and peri-normal tissues (N) was investigated via real-time PCR analysis. $(p<0.05)$. b. The expression of miR-552 in 15 pairs of ovarian cancer $(T)$ and metastasis tissues was investigated via real-time PCR analysis. $(p<0.05)$. c. The expression of miR-552 in 15 pairs of ovarian cancer $(T)$ and recurrence tissues was investigated via real-time PCR analysis. $(p<0.05)$. d. Kaplan-Meier survival curves of OS based on miR-552 expression in ovarian cancer using the online bioinformatics tool Kaplan-Meier plotter 
then counted under a microscope (Olympus) and the cell number is expressed as the average number of the cells in each field.

\section{Real-time PCR}

For detection of mature miR-552, total RNA was subjected to reverse transcription using a TaqMan MicroRNA Reverse Transcription Kit (Applied Biosystems). qRT-PCR analysis of miR-552 expression was carried out using TaqMan MicroRNA assay kits (Applied Biosystems). Results were normalized to U6 snRNA using the comparative threshold cycle $(\mathrm{Ct})$ method. The miR552 primer sequences were forward: 5' AACAGGTGAC TGGTTAGACAA 3', U6 primer sequences were forward: 5' ATTGGAACGATACAGAGAAGATT 3'.

The total cells RNA were extracted by using Trizol reagent (Invitrogen, 15596-018). Total cDNAs were synthesized by ThermoScript TM RT-PCR system (Invitrogen, 11146-057). The total mRNA amount presented in the cells was measured by RT-PCR using the ABI PRISM 7300 sequence detector (Applied Biosystems). The PTEN primer sequences were forward: 5' TCCCAGACATGACAGCCATC 3', reverse: 5' TGCTTTGAATCCAAAAACCTTACT 3 '. The $\beta$-actin was used as reference for relative expression calculation and its primer sequences were forward: $5^{\prime}$ GGCCCAGAATGCAGTTCGCCTT 3', reverse: 5' AATGGCACCCTGCTCACGCA 3 '.

\section{Western blotting assays}

Thirty micrograms of proteins were subjected to sodium dodecyl sulfate polyacrylamide gel electrophoresis and then transferred to the nitrocellulose membrane. The membrane was blocked with $5 \%$ nonfat milk and incubated with the primary antibody for $1.5 \mathrm{~h}$. The protein band, specifically bound to the primary antibody, was detected using an IRDye $800 \mathrm{CW}$ conjugated secondary antibody and LI-COR imaging system (LI-COR Biosciences). The primary antibodies were PTEN (1:1000; \# 9188, Cell Signaling Technology) and GAPDH (1:5000; \#5174, Cell Signaling Technology).

\section{Luciferase reporter assays}

The luciferase assays were carried out using the Dual-luciferase Reporter Assay System (Promega, Madison, WI, USA). Briefly, cells were co-transfected with miR-552 mimics or miR-control and pMIRreporter luciferase vector containing a specific sequence of wild-type or mutant PTEN fragment,
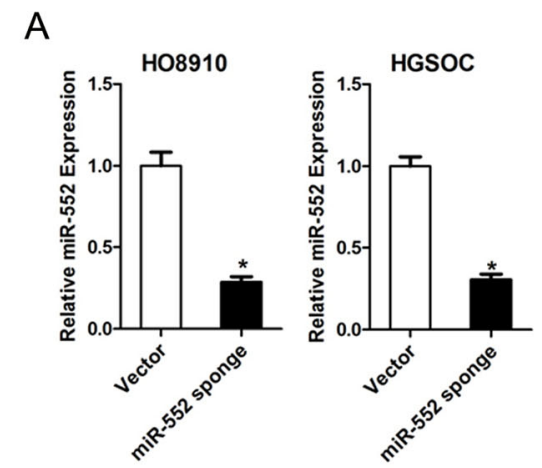

B
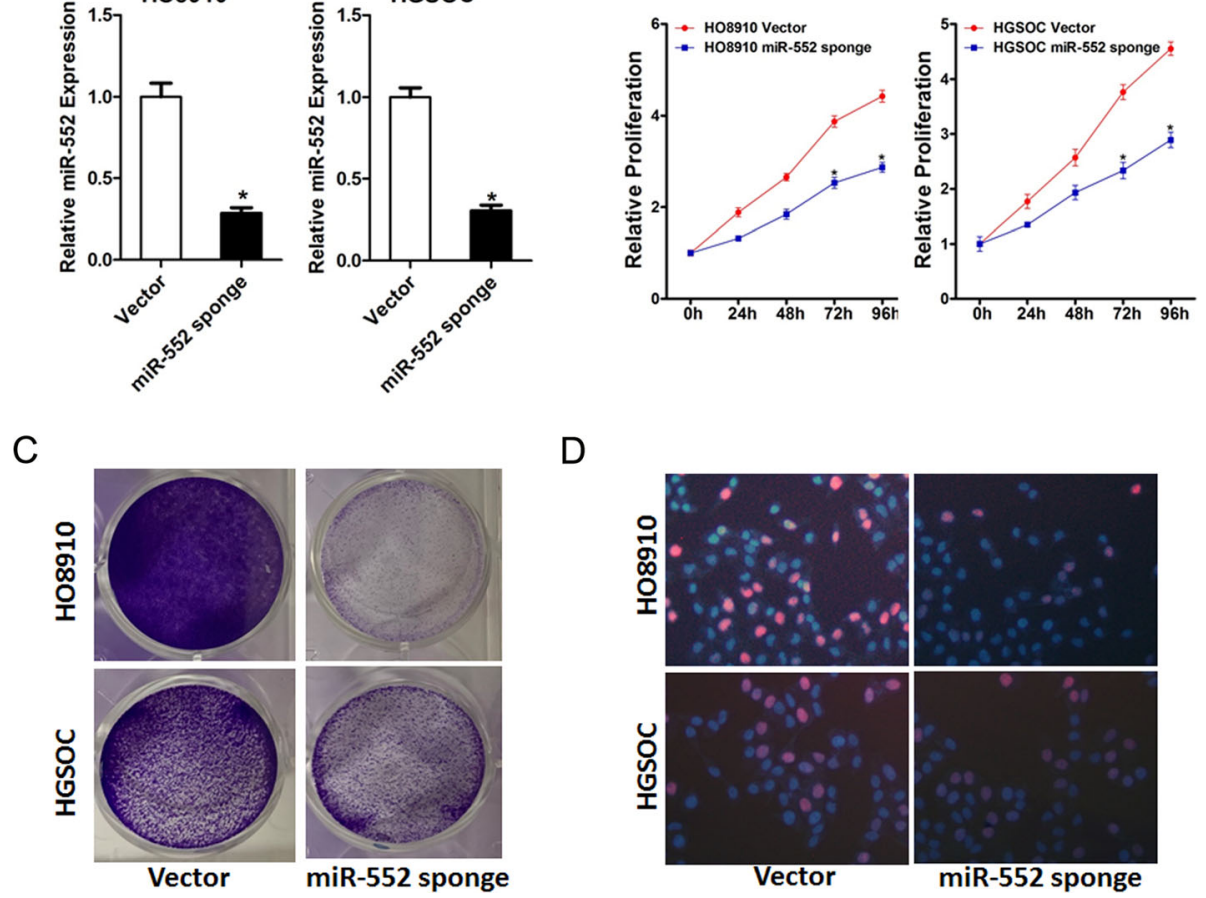

Fig. 2 Interference of miR-552 suppresses ovarian cancer cells proliferation in vitro. a. The level of miR-552 in miR-552 stably silenced HO8910 and HGSOC cells. b. Cell proliferation was measured using CCK-8 assays in HO8910 and HGSOC cells with stable depletion of miR-552. c. Colony formation assays of ovarian cancer cells with stable miR-552 sponge. $\mathbf{d}$. Cell proliferation was assessed using EdU immunofluorescence staining in HO8910 and HGSOC cells with stable interference of miR-552 
using siRNA transfection (Invitrogen, NY, USA). Cells were collected and lysed for luciferase detection $48 \mathrm{~h}$ after transfection. The relative luciferase activity was normalized against to the Renilla luciferase activity.

\section{Statistical analysis}

All statistical analyses were performed using GraphPad Prism (GraphPad Software, Inc. La Jolla, USA). Statistical analysis was carried out using $t$ test or Bonferroni Multiple Comparisons Test: $" p<0.05$. A $p$ value of less than 0.05 was considered statistically significant.

\section{Results}

Increased miR-552 expression in ovarian cancer tissues To explore the role of miR-552 in ovarian cancer progression, we measured the expression of miR-552 in a large set of human $\mathrm{OC}$ tissues. As shown in Fig. 1a, miR-552 expression was markedly elevated in OC tissues compared to paired non-tumorous tissues. We also examined miR-552 in metastasis and recurrence OC tissues, which showed that miR-552 expression was notably increased in metastasis and recurrence $\mathrm{OC}$ tissues (Fig. $1 \mathrm{~b}$ and $\mathrm{c}$ ). We further sought to determine whether upregulation of miR-552 was associated with OC patients' prognosis. Using the online bioinformatics tool Kaplan-Meier plotter [20], we found that patients with increased miR-552 expression had worse overall survival (OS) (Fig. 1d).

\section{miR-552 depletion inhibits ovarian cancer cells proliferation}

To elucidate the effect of miR-552 on ovarian cancer cells behavior, HO8910 and HGSOC cells were infected by miR-552 sponge and stable infectants

\section{A}
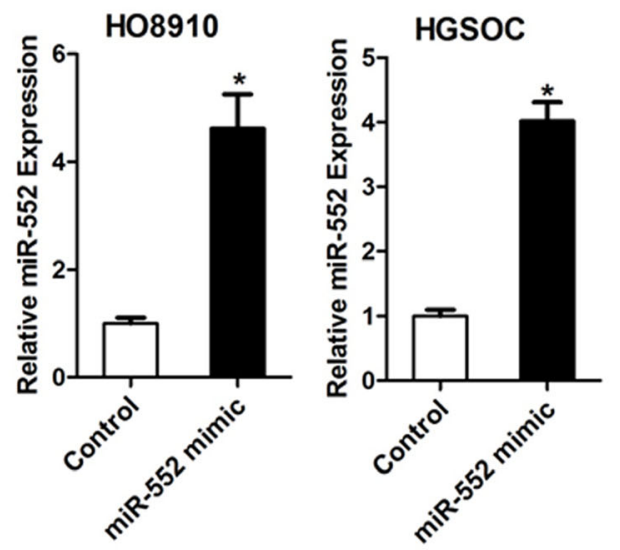

C

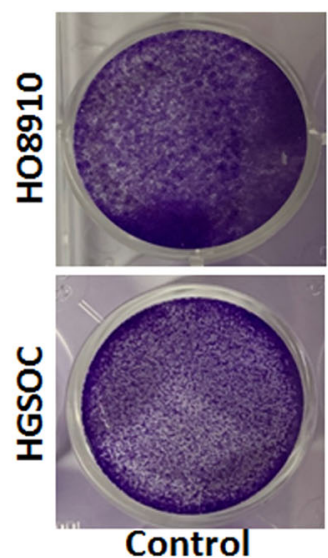

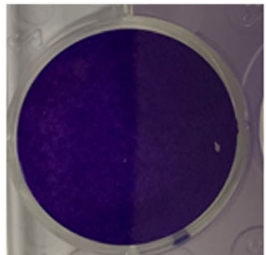

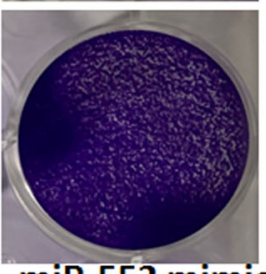

B
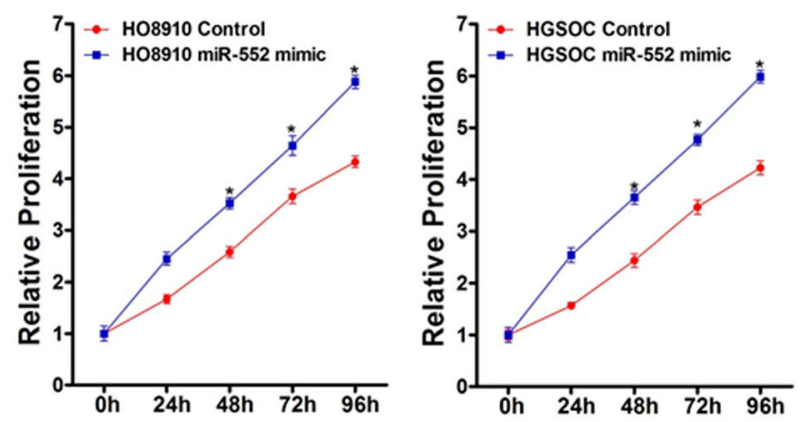

D

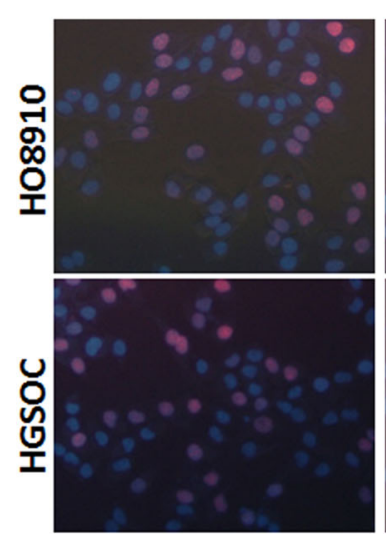

Control

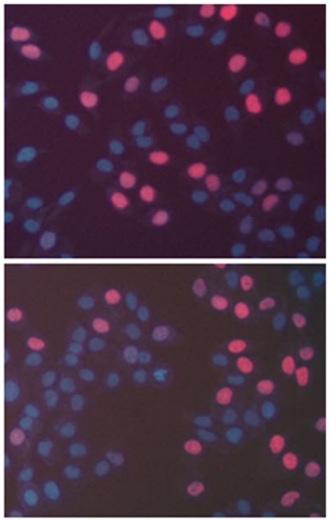

miR-552 mimic

Fig. 3 Overexpression of miR-552 facilitates ovarian cancer cells proliferation in vitro. a. The levels of miR-552 in miR-552 stably overexpressing HO8910 and HGSOC cells. b. Cell proliferations were measured using CCK-8 assays in HO8910 and HGSOC cells with stable of miR-552 mimic. c. Colony formation assays of HO8910 and HGSOC cells stably overexpressing miR-552. d. Cell proliferations were assessed using EdU immunofluorescence staining in $\mathrm{HO} 8910$ and HGSOC cells with stable of miR-552 mimic 
were established (Fig. 2a). As shown in Fig. 2b, miR-552 depletion repaired the proliferation of ovarian cancer cells markedly. In addition, ovarian cancer cells stably interfered with miR-552 sponge to form fewer and smaller colonies compared with control cells (Fig. 2c). Consistently, 5-ethynyl-2' deoxyuridine (EdU) staining confirmed that miR-552 knockdown also inhibited ovarian cancer cells growth (Fig. 2d).

\section{miR-552 overexpression promotes ovarian cancer cells proliferation}

To further confirm the effect of miR-552 on ovarian cancer cells proliferation, HO8910 and HGSOC cells were infected by miR-552 mimic and stable infectants were established (Fig. 3a). As shown in Fig. 3b, miR-552 overexpression dramatically enhanced the proliferation of ovarian cancer cells. In addition, HO8910 and HGSOC cells stably overexpressing miR-552 formed more and bigger colonies compared with their control cells (Fig. 3c). Consistently, EdU staining also confirmed that ectopic expression of
miR-552 inhibited ovarian cancer cells growth (Fig. $3 \mathrm{~d})$. Taken together, the above data showed that miR-552 promoted ovarian cancer cells growth.

\section{miR-552 knockdown inhibits ovarian cancer cells metastasis in vitro}

To explore the role of miR-552 in ovarian cancer cells metastasis, transwell assay was performed, showing that the migration ability was impaired in miR-552 inferencing ovarian cancer cells (Fig. 4a and b). In addition, matrigel invasion chamber assay revealed that miR-552 knockdown attenuated the invasiveness of ovarian cancer cells (Fig. 4c and d).

\section{miR-552 overexpression drives ovarian cancer cell transwell and invasion in vitro}

To further elucidate the role of miR-552 in ovarian cancer cells metastasis, transwell assay was performed, showing that the migration ability was enhanced in ovarian cancer cells overexpressing miR-552 (Fig. 5a and $b$ ). Furthermore, matrigel invasion chamber assay revealed that miR-552 overexpression increased the

\section{A}
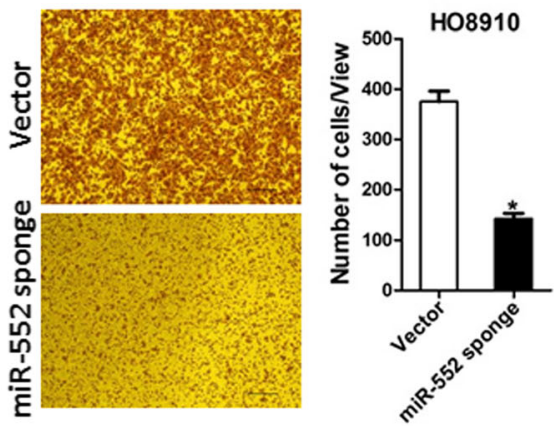

C

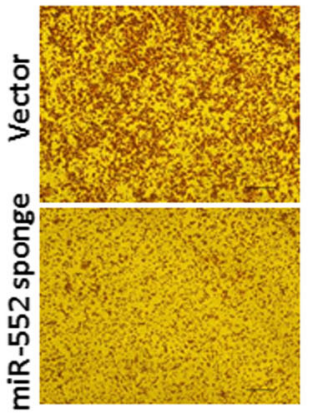

B
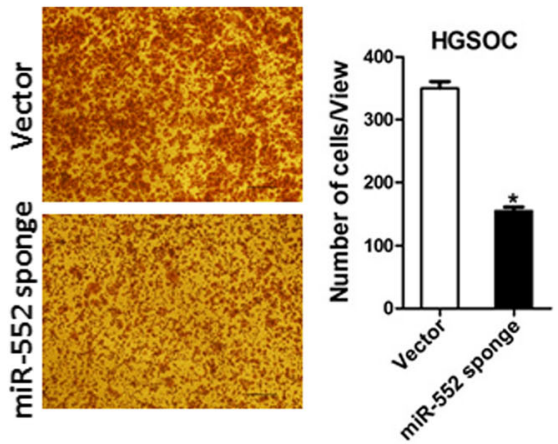

D

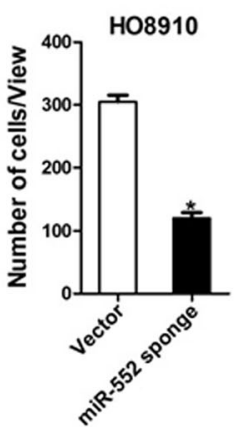

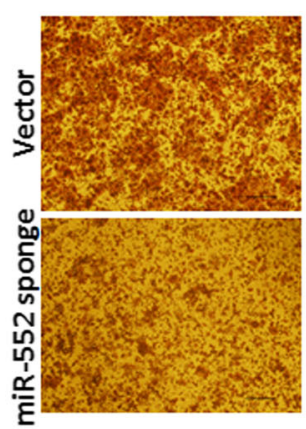

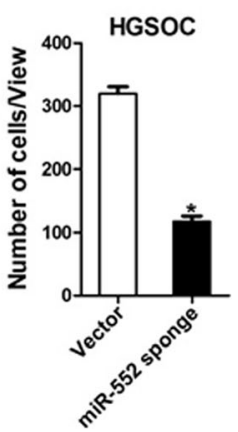

Fig. 4 miR-552 depletion suppresses ovarian cancer cells migration and invasion. a. The migration ability of HO8910 miR-552 sponge and its control cells was performed utilizing polycarbonate membrane inserts in a 24-well plate. b. The migration ability of HGSOC miR-552 sponge and its control cells was performed utilizing polycarbonate membrane inserts in a 24-well plate. c. The invasive capacity of HO8910 miR-552 sponge and its control cells were analyzed using Matrigel-coated Boyden chamber. $\mathbf{d}$. The invasive ability of HGSOC miR-552 sponge and its control cells was analyzed using Matrigel-coated Boyden chamber 
A

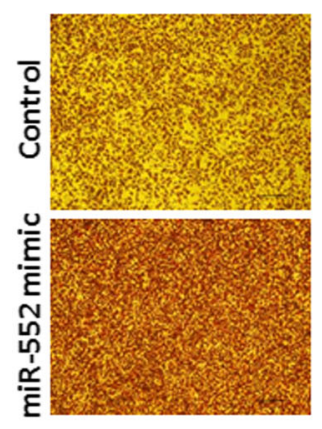

C

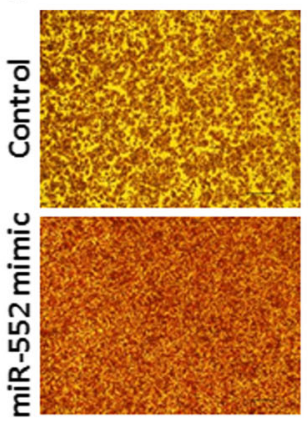

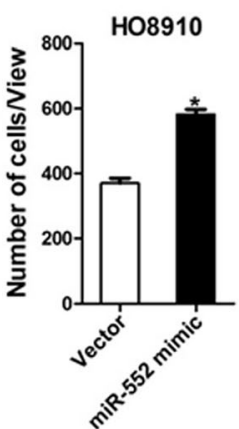

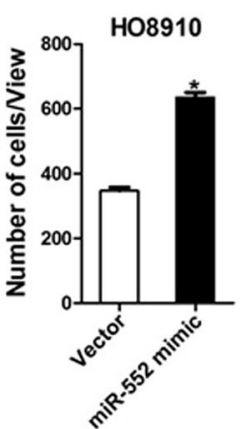

B
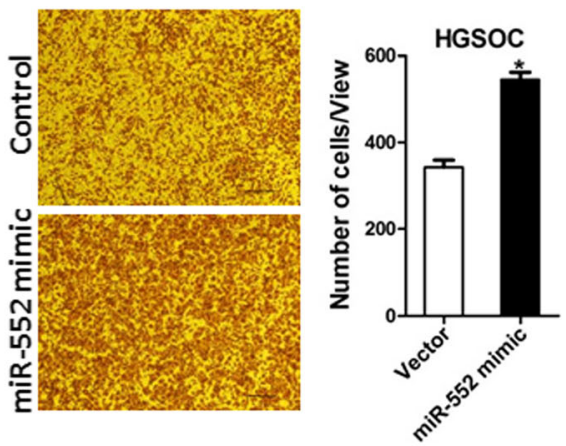

$\mathrm{D}$
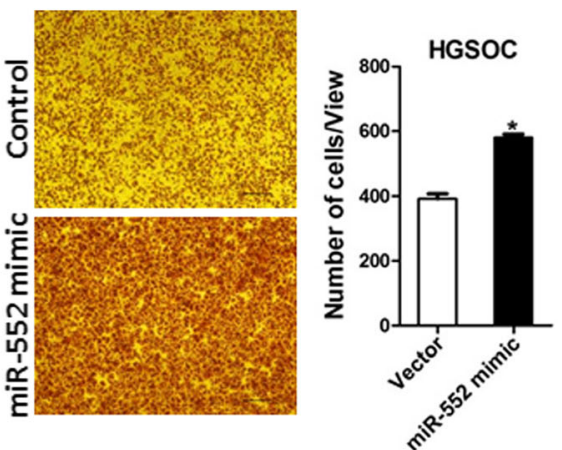

Fig. 5 miR-552 overexpression facilitates ovarian cancer cells migration and invasion. a. The migration ability of HO8910 miR-552 mimic and its control cells was performed utilizing polycarbonate membrane inserts in a 24-well plate. $\mathbf{b}$. The migration ability of HGSOC miR-552 mimic and its control cells was performed utilizing polycarbonate membrane inserts in a 24-well plate. c. The invasive capacity of HO8910 miR-552 mimic and its control cells were analyzed using Matrigel-coated Boyden chamber. $\mathbf{d}$. The invasive ability of HGSOC miR-552 mimic and its control cells was analyzed using Matrigel-coated Boyden chamber

invasiveness of ovarian cancer cells (Fig. $5 \mathrm{c}$ and d). Collectively, our results demonstrate that miR-552 disrupted the metastatic potential of ovarian cancer cells.

\section{miR-552 directly targeted PTEN to promote ovarian cancer cells progression}

Next, we attempted to identify the target genes of miR-552 that may be involved in ovarian cancer cells expansion. Bioinformatics analysis suggested that PTEN mRNA harbored a putative miR-552 binding site in its 3'-UTR (Fig. 6a). To further explore whether miR-552 directly regulates PTEN expression via interaction with its 3 '-UTR, the wild-type or mutant PTEN 3'-UTR reporter plasmids were transfected into miR-552 overexpression ovarian cancer cells and their control cells. The luciferase activity of wild-type reporter was significantly inhibited in the presence of miR-552 (Fig. 6b). However, miR-552-mediated repression of the reporter expression was abolished by mutation of the miR-552 binding site in the PTEN 3'-UTR. Moreover, PTEN mRNA and protein expression was also downregulated in miR-552 overexpression ovarian cancer cells (Fig. 6c and d). There was a significant negative correlation between miR-552 and PTEN mRNA expression in human OC tissues (Fig. 6e).

To investigate the role of PTEN in miR-552-mediated progression of ovarian cancer cells, special PTEN siRNA was used (Fig. 6f). Moreover, PTEN siRNA dramatically attenuated the distinct growth capacity between miR552 overexpression ovarian cancer cells and control cells (Fig. 6g). Consistently, PTEN siRNA also abolished the discrepancy of metastasis between miR-552 overexpression ovarian cancer cells and their control cells (Fig. 6h), suggesting that miR-552 promoted ovarian cancer cell progression by directly target PTEN pathway.

\section{Discussion}

Ovarian cancer is one of the most common tumors in the female reproductive system and has a poor prognosis, which is related to its complex pathogenesis [21]. Most ovarian cancer patients are diagnosis at late stage and always have distant metastasis [22]. The main treatment used for ovarian cancer patients include surgery, radiation therapy, chemotherapy and targeted therapy or their 
A

PTEN WT 5' ...guaguUuguacaAguguUaAaAA...3' miR-552 3' GGUUGUCCGUUUUCCAAUUUG .... 5'

PTEN Mut 5 ' ... GUAGUUUGUACAAgUaggCAAAA.... $3^{\prime}$
B

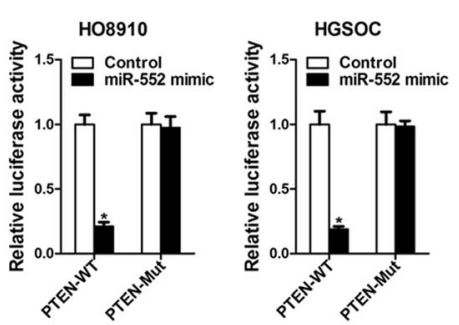

$\mathrm{E}$

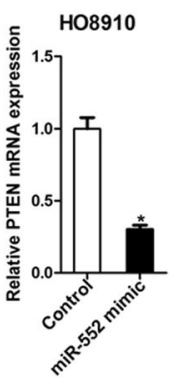

F
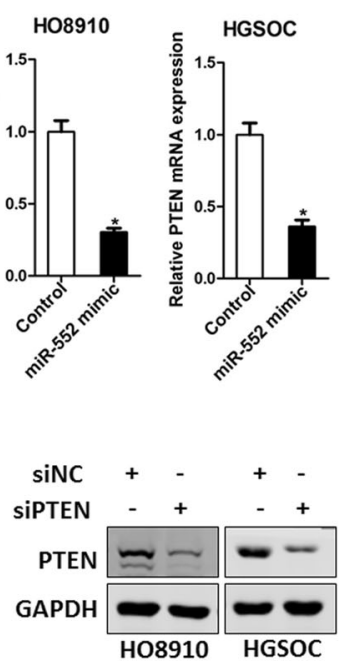

D

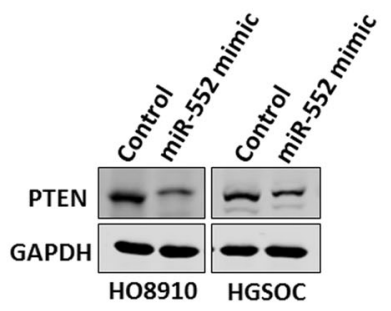

G

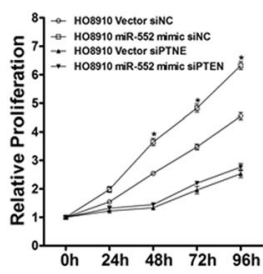

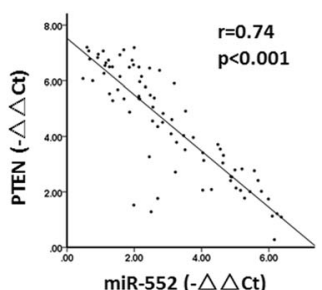

$\operatorname{miR}-552(-\triangle \triangle \mathrm{Ct})$

$\mathrm{H}$

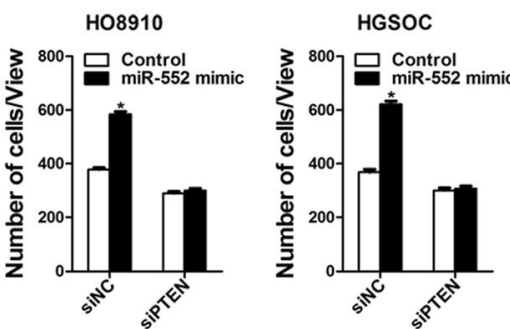

Fig. 6 PTEN was a direct target of miR-552 in ovarian cancer cells. a. A potential target site for miR-552 in the 3'-UTR of human PTEN mRNA, as predicted by the program Targetscan. To disrupt the interaction between miR-552 and PTEN mRNA, the target site was mutated. b. Luciferase reporter assays performed in HO8910 miR-552 mimic or HGSOC miR-552 mimic and their control cells transfected with wild-type or mutant PTEN 3'-UTR constructs. c. The mRNA expression of PTEN was checked in HO8910 miR-552 mimic or HGSOC miR-552 mimic and their control cells by real-time PCR. $\mathbf{d}$. The protein expression of PTEN was checked in HO8910 miR-552 mimic or HGSOC miR-552 mimic and their control cells by western blot. e. Significant correlation was observed between miR-552 and PTEN expression in human OC tissues. f. HO8910 and HGSOC cells were transfected with PTEN siRNA and then checked by western bolt assay. g. HO8910 miR-552 mimic or HGSOC miR-552 mimic and their control cells were transfected PTEN siRNA and then subjected to CCK8 assay. $\mathbf{h}$. HO8910 miR-552 mimic or HGSOC miR-552 mimic and their control cells were transfected PTEN siRNA and then subjected to migration assay

combination [1]. However, the prognosis of ovarian cancer patients is disillusionary. This was the first study to clarify that miR-552 over-expression correlated well with the poor prognosis of OC patients, suggesting that miR-552 may be a good prognostic biomarker for OC patients.

Increasing evidence has shown that miRNAs are involved in various aspects of carcinogenesis and function as either tumor suppressors or oncogenes
[23-25]. The first human disease known to be associated with miRNA deregulation was chronic lymphocytic leukemia [26]. Another role for miRNA in cancers is to use their expression level for prognosis. For instance, low miR-324a levels may serve as an indicator of poor survival in NSCLC samples [27]. Cell-free miRNA are highly stable in blood, are overexpressed in cancer and are quantifiable within 
the diagnostic laboratory. Circulating miRNAs have the potential to assist clinical decision making and aid interpretation of positron emission tomography combined with computerized tomography $[28,29]$. They can be performed at each consultation to assess disease response and detect relapse. miRNAs also have the potential to be used as tools or targets for treatment of different cancers.

It was reported that miRNAs played an important functions in ovarian cancer initiation and progression. For instance, the STAT3-miRNA-92-Wnt signaling pathway regulates spheroid formation and malignant progression in ovarian cancer [30]. miR-6126 is released via exosome and suppresses the progression of ovarian cancer cells [31]. Previous studies showed that miR-552 is upregulated in colorectal cancer tissues and promotes colorectal cancer cells progression by directly targeting DACH1. In addition, miR-552 also enhances metastatic capacity of colorectal cancer cells by targeting a disintegrin and metalloprotease 28 . However, the potential role of miR-552 in ovarian cancer has not been reported. In our above work, we found that miR-552 mimic promotes ovarian cancer cells proliferation and metastasis. Consistently, miR552 sponge inhibits ovarian cancer cells proliferation and metastasis in vitro.

PTEN acts as a famous tumor suppressor gene through the action of its phosphatase protein product [32]. PTEN's protein phosphatase activity may be involved in the regulation of the cell cycle, preventing cells from growing and dividing too rapidly [33-35]. Mutations of this gene are a step in the development of many cancers, including lung cancer, liver cancer and breast cancer [36, 37]. It also can be used as the targets for drug candidates in tumors [38]. However the exact mechanism beneath PTEN activation in ovarian cancer remains vague. We hereby revealed that miR-552 mimic downregulated PTEN mRNA and protein expression in ovarian cancer cells. Moreover, we also found that miR552 directly regulates PTEN expression via interaction with its 3'-UTR. More importantly, PTEN siRNA could abolish the distinct growth capacity or metastasis ability between miR-552 mimic ovarian cancer cells and control cells. Herein, we for first revealed that miR-552 promote ovarian cancer proliferation and metastasis via directly regulating PTEN. These findings of the present study not only shed a new light on the mechanism of ovarian cancer but suggest a potential therapeutic target against ovarian cancer patients.

\section{Conclusion}

We demonstrated for the first time that miR-552 expression is upregulated in ovarian cancer tissues, and miR-552 shRNA silencing suppresses the growth and metastasis of ovarian cancer cells. Moreover, miR-552 promoted ovarian cancer cell progression by PTEN signaling pathway. The findings of the present study not only shed new light on the mechanisms responsible for ovarian cancer progression but also suggest that miR-552 may be a novel prognostic marker and a potential therapeutic target for ovarian cancer.

\section{Supplementary information}

Supplementary information accompanies this paper at https://doi.org/10. 1186/s13048-019-0589-y.

Additional file 1: Table S1. Clinicopathological features of 80 epithelial ovarian cancer patients.

Acknowledgements

We thank Teng-Fei Zhou and Shi-Chao Li for technical assistance.

Authors' contributions

WMZ, TH, BL, QYM and HYL designed the experimental plan and wrote the manuscript with input from all other authors. TH and BL provided clinical samples and analyzed clinical data. WMZ, QYM, PHY, and HYL performed the experiments. All authors read and approved the final manuscript.

Funding

This study was funded by China Postdoctoral Science Foundation (2014 M552624), the National Natural Science Foundation of Shanghai (1438600; 19ZR1400300). The funding was used in the design of the study and collection, analysis, and interpretation of data and in writing the manuscript.

Availability of data and materials

Data generated from the study are available from the corresponding author on reasonable request.

Ethics approval and consent to participate

All procedures performed in studies involving human participants and animals were in accordance with the ethical standards of the institutional and/or national research committee and with the 1964 Helsinki declaration and its later amendments or comparable ethical standards. All of the patients provided signed informed consent. The medical ethics committee of Cao county people's hospital approved the retrieval method for cancer specimens.

Consent for publication

Agree.

Competing interests

The authors declare that they have no competing interests.

\section{Author details}

${ }^{1}$ Department of General surgery, Cao county people's hospital, East of Qinghe Road, Heze 274400, Shandong province, China. ²Department of Oncology, General Hospital of Northern Theater Command, Shenyang 110016, Liaoning Province, China. ${ }^{3}$ Department of Urology surgery, First Affiliated Hospital of Second Military Medical University, Shanghai 200433, China. ${ }^{4}$ Department of Biliary Tract Surgery, Third Affiliated Hospital of Second Military Medical University, Shanghai 200438, China. ${ }^{5}$ Department of Breast and Thyroid surgery, First Affiliated Hospital of Second Military Medical University, Shanghai 200433, China.

Received: 24 May 2019 Accepted: 4 November 2019

Published online: 09 December 2019

References

1. Marth C, Reimer D, Zeimet AG. Front-line therapy of advanced epithelial ovarian cancer: standard treatment. Ann Oncol. 2017;28:viii36-9. 
2. Maldonado L, Hoque MO. Epigenomics and ovarian carcinoma. Biomark Med. 2010;4:543-70.

3. Della Pepa C, Tonini G, Santini D, Losito S, Pisano C, Di Napoli M, Cecere SC, et al. Low grade serous ovarian carcinoma: from the molecular characterization to the best therapeutic strategy. Cancer Treat Rev. 2015;41:136-43.

4. Parkin DM, Bray F, Ferlay J, Pisani P. Global cancer statistics, 2002. CA Cancer J Clin. 2005:55:74-108.

5. Tang Z, Ow GS, Thiery JP, Ivshina AV, Kuznetsov VA. Meta-analysis of transcriptome reveals let-7b as an unfavorable prognostic biomarker and predicts molecular and clinical subclasses in high-grade serous ovarian carcinoma. Int J Cancer. 2014;134:306-18.

6. Tang H, Yao L, Tao X, Yu Y, Chen M, Zhang R, Xu C. miR-9 functions as a tumor suppressor in ovarian serous carcinoma by targeting TLN1. Int J Mo Med. 2013;32:381-8.

7. DeSano JT, Xu L. MicroRNA regulation of cancer stem cells and therapeutic implications. AAPS J. 2009;11:682-92.

8. Bayoumi AS, Sayed A, Broskova Z, Teoh JP, Wilson J, Su H, Tang YL, et al. Crosstalk between Long noncoding RNAs and MicroRNAs in health and disease. Int J Mol Sci. 2016;17:356.

9. Alberti C, Cochella L. A framework for understanding the roles of miRNAs in animal development. Development. 2017;144:2548-59.

10. Forterre A, Jalabert A, Chikh K, Pesenti S, Euthine V, Granjon A, Errazuriz E, et al. Myotube-derived exosomal miRNAs downregulate Sirtuin1 in myoblasts during muscle cell differentiation. Cell Cycle. 2014;13:78-89.

11. Li L, Tang J, Zhang B, Yang W, LiuGao M, Wang R, Tan Y, et al. Epigenetic modification of MiR-429 promotes liver tumour-initiating cell properties by targeting Rb binding protein 4. Gut. 2015;64:156-67.

12. Chen PS, Su JL, Hung MC. Dysregulation of microRNAs in cancer. J Biomed Sci. 2012;19:90.

13. Wu J, Yuan P, Mao Q, Lu P, Xie T, Yang H, Wang C. miR-613 inhibits proliferation and invasion of breast cancer cell via VEGFA. Biochem Biophys Res Commun. 2016;478:274-8.

14. Emmrich S, Streltsov A, Schmidt F, Thangapandi VR, Reinhardt D, Klusmann $\mathrm{JH}$. LincRNAs MONC and MIR100HG act as oncogenes in acute megakaryoblastic leukemia. Mol Cancer. 2014;13:171.

15. Long M, Zhan M, Xu S, Yang R, Chen W, Zhang S, Shi Y, et al. miR-92b-3p acts as a tumor suppressor by targeting Gabra3 in pancreatic cancer. Mol Cancer. 2017;16:167.

16. Cao J, Yan XR, Liu T, Han XB, Yu JJ, Liu SH, Wang LB. MicroRNA-552 promotes tumor cell proliferation and migration by directly targeting $\mathrm{DACH} 1$ via the Wnt/beta-catenin signaling pathway in colorectal cancer. Oncol Lett. 2017;14:3795-802

17. Wang J, Li H, Wang Y, Wang L, Yan X, Zhang D, Ma X, et al. MicroRNA-552 enhances metastatic capacity of colorectal cancer cells by targeting a disintegrin and metalloprotease 28. Oncotarget. 2016;7:70194-210.

18. Xiang D, Cheng Z, Liu H, Wang X, Han T, Sun W, Li X, et al. Shp2 promotes liver cancer stem cell expansion by augmenting beta-catenin signaling and predicts chemotherapeutic response of patients. Hepatology. 2017;65:1566-80.

19. Xiang DM, Sun W, Zhou T, Zhang C, Cheng Z, Li SC, Jiang W, et al. Oncofetal HLF transactivates c-Jun to promote hepatocellular carcinoma development and sorafenib resistance. Gut. 2019;68(10):1858-71.

20. Gyorffy B, Surowiak P, Budczies J, Lanczky A. Online survival analysis software to assess the prognostic value of biomarkers using transcriptomic data in non-small-cell lung cancer. PLoS One. 2013;8:e82241.

21. Zhang L, Volinia S, Bonome T, Calin GA, Greshock J, Yang N, Liu CG, et al. Genomic and epigenetic alterations deregulate microRNA expression in human epithelial ovarian cancer. Proc Natl Acad Sci U S A. 2008;105:7004-9.

22. Zhao L, Ji G, Le X, Luo Z, Wang C, Feng M, Xu L, et al. An integrated analysis identifies STAT4 as a key regulator of ovarian cancer metastasis. Oncogene. 2017;36:3384-96.

23. Jung $\mathrm{HJ}$, Suh $\mathrm{Y}$. Circulating miRNAs in ageing and ageing-related diseases. J Genet Genomics. 2014;41:465-72.

24. Filella $X$, Foj L. miRNAs as novel biomarkers in the management of prostate cancer. Clin Chem Lab Med. 2017:55:715-36.

25. Colletti M, Paolini A, Galardi A, Paolo VD, Pascucci L, Russo I, Angelis B, et al. Expression profiles of exosomal miRNAs isolated from plasma of patients with desmoplastic small round cell tumor. Epigenomics. 2019;11(5):489-500.

26. Paggetti J, Haderk F, Seiffert M, Janji B, Distler U, Ammerlaan W, Kim YJ, et al. Exosomes released by chronic lymphocytic leukemia cells induce the transition of stromal cells into cancer-associated fibroblasts. Blood. 2015;126: 1106-17.
27. Vosa U, Vooder T, Kolde R, Fischer K, Valk K, Tonisson N, Roosipuu R, et al. Identification of miR-374a as a prognostic marker for survival in patients with early-stage nonsmall cell lung cancer. Genes Chromosomes Cancer. 2011:50:812-22.

28. Mushtaq G, Greig NH, Anwar F, Zamzami MA, Choudhry H, Shaik MM, Tamargo IA, et al. miRNAs as circulating biomarkers for Alzheimer's disease and Parkinson's disease. Med Chem. 2016;12:217-25.

29. Thomou T, Mori MA, Dreyfuss JM, Konishi M, Sakaguchi M, Wolfrum C, Rao $\mathrm{TN}$, et al. Adipose-derived circulating miRNAs regulate gene expression in other tissues. Nature. 2017;542:450-5.

30. Chen MW, Yang ST, Chien MH, Hua KT, Wu CJ, Hsiao SM, Lin H, et al. The STAT3-miRNA-92-Wnt signaling pathway regulates spheroid formation and malignant progression in ovarian Cancer. Cancer Res. 2017;77:1955-67.

31. Kanlikilicer P, Rashed MH, Bayraktar R, Mitra R, Ivan C, Aslan B, Zhang X, et al. Ubiquitous release of Exosomal tumor suppressor miR-6126 from ovarian Cancer cells. Cancer Res. 2016;76:7194-207.

32. Lee EY, Muller WJ. Oncogenes and tumor suppressor genes. Cold Spring Harb Perspect Biol. 2010;2:a003236.

33. Brandmaier A, Hou SQ, Shen WH. Cell cycle control by PTEN. J Mol Biol. 2017:429:2265-77

34. Ma LL, Wang DW, Yu XD, Zhou YL. Tangeretin induces cell cycle arrest and apoptosis through upregulation of PTEN expression in glioma cells. Biomed Pharmacother. 2016;81:491-6.

35. Lu XX, Cao LY, Chen X, Xiao J, Zou Y, Chen Q. PTEN inhibits cell proliferation, promotes cell apoptosis, and induces cell cycle arrest via Downregulating the PI3K/AKT/hTERT pathway in lung adenocarcinoma A549 cells. Biomed Res Int. 2016;2016:2476842.

36. VanderLaan PA, Rangachari D, Mockus SM, Spotlow V, Reddi HV, Malcolm J, Huberman MS, et al. Mutations in TP53, PIK3CA, PTEN and other genes in EGFR mutated lung cancers: correlation with clinical outcomes. Lung Cancer. 2017;106:17-21.

37. Rangel R, Lee SC, Hon-Kim Ban K, Guzman-Rojas L, Mann MB, Newberg JY, Kodama T, et al. Transposon mutagenesis identifies genes that cooperate with mutant Pten in breast cancer progression. Proc Natl Acad Sci U S A. 2016;113:E7749-58.

38. Kim ST, Lee J, Park SH, Park JO, Park YS, Kang WK, Lim HY. Prospective phase II trial of everolimus in PIK3CA amplification/mutation and/or PTEN loss patients with advanced solid tumors refractory to standard therapy. BMC Cancer. 2017;17:211.

\section{Publisher's Note}

Springer Nature remains neutral with regard to jurisdictional claims in published maps and institutional affiliations.

\section{Ready to submit your research? Choose BMC and benefit from:}

- fast, convenient online submission

- thorough peer review by experienced researchers in your field

- rapid publication on acceptance

- support for research data, including large and complex data types

- gold Open Access which fosters wider collaboration and increased citations

- maximum visibility for your research: over $100 \mathrm{M}$ website views per year

At $\mathrm{BMC}$, research is always in progress.

Learn more biomedcentral.com/submissions 